\title{
B.М. Маракулин
}

Институт математики им. С.Л. Соболева СО РАН,

Новосибирский государственный университет, Новосибирск

\section{Контракты и доминирование В моделях конкурентной экономики}

В работе предлагается новая концепция доминирования, основанная на понятии менового (бартерного) контракта (договора). Классическое понятие коалиционного доминирования переносится на системы (сети) договоров и тем самым на отвечающие им договорные распределения (состояния), чьи свойства стабильности исследуются. Показано, что этот договорной подход әффективно моделирует условия совершенной конкуренции и в его рамках можно описать ряд известных классических понятий в совершенной модели экономики - «равновесие», «ядро», «нечеткое ядро» и проч. - в кооперативно-игровых терминах. Для несовершенных моделей, в которых не каждый контракт реализуем (разрешен), договор и договорной подход могут служить одним из первичных модельных параметров, что позволяет разрешать разнообразные теоретические проблемы.

Ключевые слова: экономика обмена, контракт, договор, договорное (контрактное) распределение, конкурентное равновесие, ядро.

JEL Classification: C62, D51.

\section{Введение}

В реальной экономике имеется множество аспектов, которыми пренебрегает классическая теория распределения ресурсов, что вызвало в 1980-х годах появление и исследование моделей несовершенных рынков. В современной экономической теории можно найти множество моделей этого типа, включающих в себя неполные (финансовые) рынки (в модель включена торговля специфическими финансовыми инструментами - активами), рынки с информационной асимметрией (о событиях будущего), секвенциальные рынки (учтен фактор времени и доверия) и т.д. ${ }^{1}$ По нашему мнению, пестрота имеющегося модельного ряда и трудности анализа многих моделей обусловлены как объективной сложностью объекта исследования (экономики), так и отсутствием унифицированного в достаточной мере инструментария исследования. Последнее влечет множественность концепций решения, в том числе имеющихся при введении понятия (коалиционного) доминирования и вытекающего из него понятия ядра экономики. Причина этого кроется в том, что, следуя классической традиции, внимание концентрируется на анализе итогового распределения ресурсов. Тот факт, что в реальной экономической системе это распределение является итогом множества сделок обмена между группами экономических агентов (коалициями), обычно остается без внимания. Причем не каждая сделка (обмен) осуществима в реальной экономике, чему может быть множество причин, выраженных на институциональном, физическом, информационном и прочих уровнях (этическом, поведенческом). Мы считаем, что фокус теории должен

$\overline{1}$ Примеры моделей несовершенных рынков можно найти в работах из представленного в конце статьи списка литературы. 
быть скорректирован и сконцентрирован собственно на сделках по обмену продуктами - контрактах или договорах, - которые должны составлять элементную базу теоретических построений (наряду с другими элементами модели), т.е. контракт по обмену товарами должен стать основным модельным инструментом, применяемым вместо распределений.

В данной работе предлагается использовать договорной подход как инструмент для экономического моделирования, который уже сейчас способен прояснить и разрешить многие трудные вопросы экономической теории, появляющиеся при анализе несовершенных рынков (например, неполные рынки и модели с информационной асимметрией). Этот подход предоставляет иной взгляд на условия совершенной конкуренции и продуктивно работает в анализе доминирования и ядра, применяемых в экономических моделях разного вида. Более того, этот подход может помочь при анализе рыночных процессов в классических модельных рамках, например, он обеспечивает лучшее понимание процессов нащупывания равновесия, являясь при этом избавленным от идеи аукционера, управляющего динамикой изменения неравновесных цен (предполагается в классическом нащупывании, см., например, (Arrow, Hahn, 1991).

Первые попытки ввести формальное понятие (бартерного) контракта в контексте стандартной модели обмена были предприняты в работах (Полтерович, 1970; Макаров, 1980, 1982; Козырев, 1982, 1981). В работе (Полтерович, 1970) исследовались некоторые виды договорных процессов без разрыва договоров и анализировались свойства распределений, к которым эти процессы сходятся. Общая идея договорного подхода в виде специфического терминологического языка была предложена В.Л. Макаровым (Макаров, 1980, 1982). А.Н. Козырев $(1981,1982)$ впервые рассмотрел частичный разрыв договоров, что повлекло изучение соответствующих типов устойчивости, и на этом пути были получены новые позитивные результаты. Основные результаты настоящей работы являются авторскими и вполне оригинальными ${ }^{2}$. Терминология также новая и охватывает существенно более широкий спектр специфических договорных понятий по сравнению с работами В.Л. Макарова и А.Н. Козырева (изменилось само понятие контракта), причем нет совпадения даже в части аналогичных по смыслу понятий. В западной литературе теория бартерных договоров в своем специфическом виде не имела развития. О других имеющихся в научной литературе близких подходах будет сказано ниже.

В стандартной модели обмена бартерный контракт - это любой допустимый элементарный обмен продуктами среди потребителей. Контракты можно складывать, и любому (конечному) множеству договоров можно сопоставить итоговое распределение продуктов среди экономических агентов - как результат суммирования договоров и «начального» распределения. Причем предполагается, что достижи-

${ }^{2}$ Для полноты изложения в работу были включены некоторые известные ранее (несложные) первичные факты и утверждения, там, где это нужно, приведены необходимые ссылки. 
мые множества (допустимых) договоров (назовем их «сетями контрактов») могут изменятся в течение экономической жизни. Любой экономический агент или их коалиция может разрывать контракты, в которых участвует, а коалиция агентов - заключать новые контракты.

Более того, если это им выгодно, потребители могут частично разрывать контракты (подписанные в прошлом). Частичный разрыв контракта означает его замещение контрактом меньшего объема с теми же пропорциями обмена. Это приводит к концепции правильно договорного распределения и приближает контрактные процессы к рыночным в условиях совершенной конкуренции. Распределение называется правильно договорным, если оно реализуемо некоторой сетью договоров, стабильной относительно как заключения новых договоров, так и частичного разрыва уже имеющихся. В то же время распределения из ядра описываются как договорнъе распределения. Это распределения, которые можно реализовать сетью договоров, стабильной относительно процедур (полного) разрыва договоров и заключения новых договоров. Таким образом, единственное различие между двумя понятиями договорного распределения состоит в том, что в первом случае допускается частичный разрыв договоров, а во втором - разрыв (отказ от контракта) возможен только полностью. Таким способом, не прибегая к стоимостным категориям, можно охарактеризовать не только ядро, но и равновесие - как правильно договорное распределение (предположения: внутренняя точка, гладкие полезности). Математическая природа этого феномена подобна тому, как равновесие Эджворта ${ }^{3}$ (или элементы нечеткого ядра) становится конкурентным равновесием в обычном смысле, но именно это и является классическим способом моделирования условий совершенной конкуренции.

В работе развивается теория договоров сначала в рамках абстрактной модели экономики, которая затем применяется к классическим рынкам. Рассматриваются и изучаются формальные правила оперирования с множествами допустимых договоров. Различия в этих правилах соответствуют различиям в типах стабильности сетей и реализуемых ими распределений. Виды этих «стабильностей», совместно со свойством допустимости контрактов из данной сети, отражают различные поведенческие, физические и институциональные принципы, формально заданные в теоретико-игровой форме, которые имеются в реальной жизни и неоклассической экономической теории. Итак, различным видам стабильности сети соответствуют разные виды договорного или контрактного распределения, а также их модификации, которые могут ослабить или усилить эту стабильность. В работе вводится новый формализм и ряд новых оригинальных договорных понятий: «когерентные», «совершенные», «нечеткие» и «сложно договорные» распределения; раскрываются имеющиеся между ними соотношения. Таким способом, с учетом структуры допустимых контрактов, можно описать многие хорошо известные в экономической тео-

${ }^{3}$ Это распределение, которое, будучи реплицированным нужное число раз, попадает в ядро каждой из реплик исходной модели. 
рии понятия - такие как «ядро», «конкурентное равновесие», «граница Парето» и т.д. - в терминах стабильных сетей контрактов. В работе выявляется взаимосвязь между элементами нечеткого ядра и так называемыми нечетко договорными распределениями, что важно само по себе: так, нечеткое ядро получает естественную (новую) интерпретацию, которая, в частности, может применяться в бесконечномерных моделях (например, чтобы установить существование равновесий, - как это сделано в (Marakulin, 2006), где обобщаются результаты из (Florenzano, Marakulin, 2001)).

Недопустимость некоторых договоров является специфической чертой многих современных неклассических моделей, и здесь концентрируется суть договорного подхода. Однако в данной работе будет рассмотрено только первое возможное приложение, а договорная экономика изучена без ограничения на допустимость договоров, что соответствует случаю классических рынков. Я надеюсь, что читатель увидит самостоятельно потенциал договорного подхода для анализа действительно несовершенных экономик (например, неполные (Маракулин, 2003; Magill, Quinzii, 2002) и секвенциональные рынки (Gale, 1978; Repullo, 1988), рынки с информационной асимметрией (Schwalbe, 1999; Aliprantis, 2001; Glycopantis, Yannelis, 2004; Маракулин, 2009; и т.д.). В несовершенных экономиках ограничения на допустимость договоров обычно воплощаются в виде требования принадлежности к некоторому подпространству всех бартерных договоров. Это может быть подпространство функций, измеримых относительно информационной структуры (алгебры), для моделей с информационной асимметрией, или специфическое подпространство, образованное посредством реальных активов в неполных рынках (так как прямой обмен продуктами между разными состояниями мира невозможен), и т.д.

В чем состоит основное теоретическое значение договорного подхода? Главное отличие в том, что, будучи основанным на расширенных представлениях о коалиционной стабильности распределения ресурсов, этот подход позволяет определить равновесие в микротерминах и тем самым открыть принципиальную возможность описания процесса экономического взаимодействия при достижении равновесия (вместо нащупывания, которое локальные взаимодействия не описывает). Ключевую роль здесь играет возможность разрыва договоров, причем разрыва частичного и даже асимметричного (на стадии планирования новых сделок). Посредством введения частичного разрыва договоров в модели задаются условия совершенной конкуренции, при этом собственно метод ее моделирования гораздо проще известных в литературе. Действительно, в классической теории общего равновесия условия совершенной конкуренции (см., например, (Vind, 1995)) описываются как наличие континуума агентов, каждый из которых пренебрежимо мал по отношению к эко- 
номике в целом (задано неатомическое пространство с мерой, описывающее множество всех агентов (Aumann, 1964). Далее доказывается теорема о совпадении ядра и равновесия, теорема весьма нетривиальная, применяющая теорию интегрирования точечно-множественных отображений (см. также (Hildenbrand, 1974)). Известны и более утонченные методы моделирования совершенной конкуренции, в том числе использующие нестандартный анализ: изучаются экономики, в которых число агентов гиперконечное (Anderson, 1992). Однако нужна ли в действительности столь сложная и продвинутая математика, чтобы обосновать совершенную конкуренцию? С точки зрения договорного подхода в этом нет нужды, и все, что требуется, это учесть в модели возможность иастичного разрыва договоров. Именно в этом состоит содержательный смысл доказанных во второй части работы теорем 2,3 , а также других не вошедших в работу характеризаций договорных распределений разного рода, позволяющих заключить их равновесные свойства. Более того, теперь равновесное распределение и распределения из ядра становятся объектами одного порядка: реализуются сетью договоров, стабильной в специфическом смысле, причем стабильность равновесия заведомо более сильная. Собственно частичный разрыв договоров можно интерпретировать разными способами, один из которых состоит в том, что таким путем неявно учитывается динамическая составляющая договорного процесса (за кадром модели). Именно с течением времени рациональный индивид приобретает опыт и учится не повторять ошибочные (невыгодные) для него договоры, повторно реализуя заключенные в прошлом контракты в оптимальном (на текущий момент) для себя объеме (пропорции обмена сохраняются). Если же ни у кого нет желания частично рвать договоры, а также заключать новые, это означает, что экономическая система в целом вышла на динамически стабильный режим функционирования. Таким образом, допуская частичный разрыв договоров, мы как бы позволяем индивиду конкурировать самому с собой - реализации того же агента в разные моменты времени, - а также с разными другими реализациями прочих индивидов.

Отметим, что изложенная теория не связана непосредственно с теорией экономических торгов (bargainings, см., например, (Muthoo, 1999)) и рассматриваемых в ее контексте решений (Нэша и др.). Здесь также не изучаются какие либо процедуры переговорного процесса, выводящие агентов на оптимальный (справедливый) договор. Однако представляется, что такого рода результаты могут быть включены в теорию бартерных договоров (хотя бы частично) в последующих исследованиях.

В западной литературе также представлено направление, в котором изучаются «игры, в которых индивиды встречаются и торгуются» (DMBG-игры). Игры этого типа применяются к моделям экономики типа Эрроу-Дебре и имеют близкий к договорному подходу идеологи- 
ческий контекст. В наиболее известном варианте (Gale, 2000) рассматривается экономика с континуумом агентов, представленных конечным числом типов. В каждый момент времени (дискретно и совпадает с натуральным рядом) каждый агент может встретиться с партнером с некоторой (фиксированной) вероятностью. После встречи пары агентов равновероятно выбирается агент, который делает предложение другому агенту, желая получить продуктовый вектор $w \in \mathbb{R}^{l}$. Если последний агент принимает предложение, то его потребительский набор изменится на $-w$, а у его партнера на $+w$. В договорной терминологии $w$ - это предложение заключить меновый контракт вида $(w,-w)$. Если агент не отвергает предложение, то потребительские наборы остаются неизменными. Индивид, не принявший сделанное ему предложение (и никакой другой), может в следующий момент уйти с рынка. Агент,

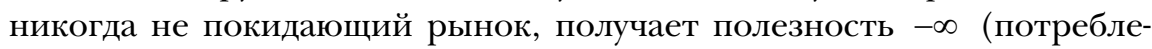
ние возможно только после ухода). Стратегия игрока - это некий план действий, описывающий его поведение в разных торговых ситуациях, в зависимости от текущего потребления, типа партнера, его текущего потребления и сделанного им предложения (если так случится). Стратегия зависит от совершенных ранее индивидом сделок и, если ему сделано предложение, может принимать значения: «принять предложение», «отклонить предложение и остаться на рынке», «отклонить предложение и уйти с рынка».

Для этой DMBG-игры рассматриваемое стратегическое равновесие представляет собой специализированный вид совершенного в подыграх байесовского равновесия в рамках соответствующей игры в развернутой форме. Основным результатом исследования является теорема, в которой утверждается, что в каждом стратегическом равновесии игрок покидает рынок с вероятностью 1, если его потребительский набор является набором, отвечающим вальрасовскому равновесию. Таким образом, данное направление предлагает специфический ответ на вопрос о том, как и почему экономическая система достигает конкурентного равновесия.

В литературе имеется множество обобщений данного подхода, см., например, (Dagan, Serrano R., Volij, 2000; Yildiz, 2003). Наконец отметим, что, сравнивая стратегический поход с договорным, можно заметить заключенную в основном результате возможность разрыва договоров - ключевой элемент договорного подхода - игроки следуют равновесным стратегиям, в которых применяются контракты, прошедшие селективный отбор, т.е. неприемлемые варианты были (где-то в предыстории равновесия) отбракованы (разорваны!). Конечно, в договорном подходе не представлена такая широкая картина возможных вариантов неравновесного взаимодействия, так как это сделано при стратегическом подходе. Однако это само по себе не является недостатком, так как на первый план выходит кооперативная составляющая итогового распределения, представленная в агрегированном 
виде посредством стабильной системы договоров. Более того, в отличие от стратегического подхода в рамках договорного процесса (Mаракулин, 2006) экономика функционирует и проходит через множество неравновесных стадий и только в пределе выходит на стабильный равновесный режим, что остается за пределами стратегической модели. Кроме того, по сравнению со стратегической договорная модель гораздо проще и лучше поддается анализу.

Работа организована следующим образом. В разд. 1 вводится модель, даются основные определения и предположения в общих рамках абстрактной договорной экономики обмена. В разд. 2 изучается стандартная экономика чистого обмена как первое возможное приложение нашего подхода: исследуются свойства введенных договорных понятий и изучается их взаимосвязь с известными в теории общего равновесия.

\section{1. Модель договорной экономики обмена}

Рассмотрим обычную модель экономики обмена. Пусть в модели $E$ обозначает (конечномерное) пространство продуктов и имеется (конечное) множество агентов (торговцев или потребителей) $\mathcal{I}=\{1, \ldots, n\}$. Потребитель $i \in \mathcal{I}$ стандартным образом характеризуется собственным потребительским множеством $X_{i} \subset E$, вектором исходных запасов $\omega_{i} \in E$ и отношением предпочтения, описанным в виде точечно-множественного отображения $\mathcal{P}_{i}: X_{i} \Rightarrow X_{i}$, где множество $\mathcal{P}_{i}\left(x_{i}\right)$ содержательно означает совокупность всех потребительских наборов, строго предпочитаемых агентом $i$ набору $x_{i}$. Будет использоваться также обозначение $y_{i} \succ_{i} x_{i}$, которое по определению эквивалентно $y_{i} \in \mathcal{P}_{i}\left(x_{i}\right)$. Таким образом, экономика чистого обмена может быть представлена в виде тройки

$$
\mathcal{E}=\left\langle\mathcal{I} \mathcal{I}, E,\left(X_{i}, \mathcal{P}_{i}, \omega_{i}\right)_{i \in \mathcal{I}}\right\rangle
$$

Пусть $L=E^{\mathcal{I}}$ - пространство состояний. Обозначим через $\omega=\left(\omega_{i}\right)_{i \in \mathcal{I}} \in L$ вектор исходных запасов (всех) торговцев рассматриваемой модели, положим $X=\prod_{i \in I} X_{i}$, и пусть

$$
\mathcal{A}(X)=\left\{x \in X \mid \sum_{i \in \mathcal{I}} x_{i}=\sum_{i \in \mathcal{I}} \omega_{i}\right\}-
$$

множество всех достижимых распределений (состояний) модели $\mathcal{E}$.

В дальнейшем всегда предполагается, что модель $\mathcal{E}$ удовлетворяет следующему предположению.

Предположение 1. Для каждого $i \in \mathcal{I}:$ множество $X_{i}$ выпуклое, телесное и замкнутое, вектор $\omega_{i} \in X_{i}$ и для каждого $x_{i} \in X_{i}$ существует открытое выпуклое $G_{i} \subset E$ такое, ито $\mathcal{P}_{i}\left(x_{i}\right)=G_{i} \cap X_{i}$ u $x_{i} \in \overline{\mathcal{P}_{i}\left(x_{i}\right)} \backslash \mathcal{P}_{i}\left(x_{i}\right)^{4}$ nрu $\mathcal{P}_{i}\left(x_{i}\right) \neq \varnothing$.

Заметьте, что в силу предположения 1 насыщаемые предпочтения допустимы, однако при $\mathcal{P}_{i}\left(x_{i}\right) \neq \varnothing$ предпочтения локально ненасыщенные в точке $x_{i}$.

\footnotetext{
${ }^{4}$ Символом $\bar{A}$ обозначено замыкание множества $A$, а «\» означает теоретико-множественную разность.
} 
В рамках модели $\mathcal{E}$ можно рассмотреть формальный механизм заключения, разрыва и перезаключения договоров (который можно назвать договорным механизмом). Этот механизм отражает идею того, что (любая) группа агентов способна реализовывать (допустимые) обмены продуктами между членами этой группы (коалиции), которые называются контрактами, и определяет правила оперирования с их множествами (договоров).

Формально любое перераспределение продуктов $v=\left(v_{i}\right)_{i \in I} \in L$, где $v_{i} \in E, i \in \mathcal{I}$, т.е. любой вектор $v \in L$, удовлетворяющий $\sum_{i \in I} v_{i}=0$, называется контрактом.

Однако не всякое перераспределение можно реализовать в экономике - существуют институциональные, физические и поведенческие ограничения в моделях экономики разного вида. По этой причине абстрактная модель договорной экономики, в дополнение к стандартной структуре экономики обмена, оснащается также новым элементом - множеством допустимых (разрешенных) договоров $\mathcal{W} \subset L$. Таким образом, договорная экономика (обмена) может быть в краткой форме описана как четверка $\mathcal{E}^{c}=\left\langle\mathcal{I}, E, \mathcal{W},\left(X_{i}, \mathcal{P}_{i}, \omega_{i}\right)_{i \in \mathcal{I}}\right\rangle$.

В дополнение к предположению 1 в общем случае постулируется еще одно предположение.

Предположение 2. Множество $\mathcal{W}$ является звездным в нуле в $L$, m.e. $v \in \mathcal{W} \Rightarrow \lambda v \in \mathcal{W} \quad \forall 0 \leq \lambda \leq 1$.

Назовем модель экономики гладкой, если для каждого $i \in \mathcal{I}$

$$
\mathcal{P}_{i}\left(x_{i}\right)=\left\{y \in X_{i} \mid u_{i}(y)>u_{i}\left(x_{i}\right)\right\} \quad \forall x_{i} \in X_{i}
$$

для некоторой дифферениируемой квазивогнутой функции $u_{i}$, определенной на (некоторой) открытой окрестности множества $X_{i}$.

В рамках договорной экономики нас могут интересовать только такие множества контрактов, которые реализуют достижимъе распределения и которые допускают такой вид операций с этим множеством как разрыв (любой) части договоров. Сказанное мотивирует следующее важное определение.

Конечная совокупность $V$ допустимых контрактов называется сетью контрактов (относительно $\omega \in \mathcal{A}(X))$, если $\omega+\sum_{v \in U} v \in X \quad \forall U \subseteq V$.

Формализуем далее операции разрыва существующих и заключения новых договоров. Для любого контракта $v \in V$ положим: $S(v)=\operatorname{supp}(v)=\left\{i \in \mathcal{I} \mid v_{i} \neq 0\right\}-$ носитель контракта $v$. Предполагается, что любой контракт $v \in V$ может быть разорван (исключен из сети) любым торговцем из $\mathcal{S}(v)$, так как он может не выполнить своих обязательств. Кроме того, любая непустая группа (коалиция) потребителей может заключить (подписать) новый контракт. Будучи рассмотрены совместно, т.е. как одновременная процедура, эти операции позволяют коалиции $T \subset \mathcal{I}$ создавать новые сети контрактов. Условимся обозначать множество всех таких сетей символом $F(V, T)$. При этом 
с формальной точки зрения постулируется, что каждый элемент $U \in F(V, T)$ удовлетворяет следующим требованиям:

i) $v \in V \backslash U \Rightarrow \mathcal{S}(v) \cap T \neq \varnothing$;

ii) $v \in U \backslash V \Rightarrow \mathcal{S}(v) \subseteq T$.

Условие $i$ ) означает, что только члены $T$ способны разрывать контракты из $V$, условие $i$ ) - что только члены $T$ могут подписывать новые контракты.

Заметьте, что в силу изложенных требований и по определению сети договоров (контрактов) коалиция может рвать любые подмножества договоров данной сети, лишь бы было выполнено требование $i$ ). В противном случае - имея дело с произвольными наборами договоров - коалиция должна была бы учитывать возможность реализации недопустимого распределения, что инициирует разрыв других договоров не-членами коалиции. Тем самым последствия разрыва становятся плохо предсказуемыми и, главное, неоднозначно прогнозируемыми для членов коалиции, что приводит к концептуальным затруднениям.

Распространим доминирование по коалиции на сети договоров. Это свойство, записанное как $U_{T} V$ ( $U$ доминирует $V$ по коалиции $T$ ), означает, что

i) $U \in F(V, T)$;

ii) $x_{i}(U) \succ_{i} x_{i}(V)$ для всех $i \in T$.

Определение 1. Сеть контрактов $V$ называется стабильной, если не существует сети $U$ и такой коалиции $T \subset \mathcal{I}, T \neq \varnothing$, что $U \succ_{T} V$.

Распределение $x$ называется договорным, если $x=x(V)$ для некоторой стабильной сети $V$.

Стабильность сети может быть как ослаблена, так и усилена, наиболее важные из имеющихся возможностей описываются ниже.

Определение 2. Сеть контрактов $V$ называется стабильной снизу, если нет такой сети $U$ и коалиции $T \subset \mathcal{I}, T \neq \varnothing$, что $U \succ_{T} V$ и $U \subset V$.

Сеть контрактов $V$ называется стабильной сверху, если нет такой сети $U$ и коалиции $T \subset \mathcal{I}, T \neq \varnothing$, что $U \succ_{T} V$ и $V \subset U$.

Сеть контрактов, стабильная сверху и снизу, называется слабо стабильной.

Распределение $x$ называется договорным снизу, сверху или слабо договорным, если $x=x(V)$ для некоторой стабильной снизу, сверху или слабо стабильной сети $V$, соответственно.

Должно быть понятно, что все данные понятия стабильности и доминирования могут применяться в стиле - «относительно данного достижимого распределения» - это распределение используется вместо вектора исходных запасов (слабо договорного распределения) действительно слабее, чем соот- 
ветствующее понятие стабильной сети (договорного распределения). Разница состоит в том, что в первом случае операции разрыва и заключения новых договоров применяются раздельно, а во втором случае - одновременно. Ниже, в рамках модели рынка, будут рассмотрены соотношения между множествами договорных, снизу, сверху и слабо договорных распределений, они соответствуют хорошо известным в экономической теории понятиям.

Как будет протекать процесс заключения новых и разрыва имеющихся договоров? Предположим, что идет что-то вроде процесса нащупывания (кооперативный tâtonnement), который, например, может протекать следующим образом. Для простоты представим себе, что имеется упорядоченный список коалиций. На первом этапе (итерации) коалиции в указанном порядке начинают заключать новые контракты и /или рвать имеющиеся (переходя к сетям из $F\left(V_{\xi}, T_{\xi}\right)$, где $\xi$-номер коалиции). Причем первая коалиция «стартует» с исходного распределения ресурсов $\omega$ и, так как до нее контрактов не заключалось, с сети $V_{1}=\varnothing$. Этап заканчивается, когда коалиция с наибольшим номером сделала свой выбор. Далее начинается второй этап, где происходит то же, что и на первом, но при условии, что первая в списке коалиция имеет дело с сетью договоров, сложившейся в конце первого этапа. Неподвижные точки такого итерационного процесса в точности отвечают договорным распределениям и устойчивым системам договоров. Ясно, что порядок «появления на арене» какой-либо коалиции в рамках одного этапа не является существенным фактором. Более того, данная коалиция может «появляться» несколько раз в течение одной итерации, и этот порядок может меняться от одного этапа к другому. Действительно важно только то, чтобы каждая коалиция имела шанс появиться в течение бесконечного числа итераций. В целом сценарий не предполагает каких-либо временных рамок продолжительности итераций, а их общее число потенциально неограниченно (тем самым итерация длится бесконечно малое время). На неформальном содержательном уровне мы предполагаем, что процесс закончится за «разумное время», и экономика в целом перейдет в некоторое стационарное состояние. Именно эти потенциально возможные стационарные состояния и являются предметом нашего внимания.

Отметим, что если в рассматриваемом итерационном процессе, начиная с некоторой итерации (кроме первой), запретить коалициям заключать новые договоры, то таким образом можно реализовать стационарные состояния и сети договоров, устойчивые снизу (в одном из вариантов, описанных в работе, - это зависит от того, какой тип разрыва договоров допустим, - можно рвать только полностью, частично или с переходом к эквивалентным). Аналогичным образом, запрещая разрыв контрактов или действуя в смешанном режиме (через итерацию - запрет на подписание нового контракта, затем запрет на разрыв и т.д.), можно реализовать устойчивые сверху и слабо устойчивые договорные распределения соответственно. 
Замечание. Подобные представления на процессы продуктового обмена, иногда называемые бартерными процессами, можно найти в работах (Полтерович, 1970; Madden, 1975; Graham et al., 1976) и др. Однако в этих и подобных работах собственно договорной подход не разрабатывался, а изучались другие специфические вопросы.

Продолжим далее список различных видов стабильности и их модификаций, усиливая стабильность в отношении процедуры разрыва договоров. Ясно, что сеть, которая не является стабильной снизу, не может сколь-нибудь долго существовать в реальной экономике. По этой причине внимание в последующих рассмотрениях будет ограничено только стабильными снизу сетями. Прежде всего введем отношение эквивалентности на множестве всех таких сетей, это отношение позволяет частично делить контракты. Чтобы определить его, сначала рассмотрим специфическое отношение частичного порядка. Это упорядочивание определяется по правилу:

$$
U \geq V \Leftrightarrow \exists \text { отображение "на" } f: U \rightarrow V:
$$

i) $\lambda f(u)=u$ для некоторого $0 \leq \lambda \leq 1$ и каждого $u \in U$;

ii) $\sum_{u \in f^{-1}(v)} u=v$ для каждого $v \in V$.

Из определения легко увидеть, что сеть $U$ состоит из (конечного) разбиения контрактов из $V$ (здесь $f^{-1}(v)$ является разбиением контракта $v$ ). Минимальные элементы в смысле данного отношения порядка в множестве всех сетей называются корневыми. Отношение эквивалентности может быть теперь введено следующим образом:

$$
U \simeq V \Leftrightarrow \exists \text { сеть } W: V \geq W \& U \geq W .
$$

Запись $U \simeq V$ означает, что эти сети имеют общий корень.

Определение 3. Распределение $x$ называется правильно договорным (соответственно правильно договорным снизу, слабо правильно договорным), если существует сеть $V$ такая, что $x=x(V)$ и для каждого $U \simeq V$ распределение $x=x(U)$ является договорным (соответственно договорным снизу, слабо договорным).

Как уже отмечалось выше, экономическое значение правильно договорного распределения состоит в том, что наряду с возможностыю заключать новые контракты агенты могут частично рвать старые контракты (в одновременном или раздельном (для «слабых») стиле). Простейший способ определить стабильность такого рода - это потребовать, чтобы любая сеть вида $\alpha V \cup(1-\alpha) V$ была стабильной при любом $\alpha \in[0,1]$. В целом правильно договорные понятия можно интерпретировать двумя способами. Во-первых, в поведенческих терминах агенты предпочитают заключать много контрактов малого объема, вместо того чтобы заключить один «большой» контракт. Таким способом они обретают больше әкономической свободы, с тем чтобы при необходимости разорвать часть контрактов. Вторая возможность - это трактовать правильный контракт как предварительное соглашение. В соглашении этого типа задаются жестко 
только пропорции обмена, в то время как объем контракта является величиной гибкой и окончательно фиксируется только в конце договорного процесса. Из сказанного должно быть понятно, что свойство распределения быть стабильным (в одном из смыслов) существенно усиливается, когда к термину добавляется прилагательное «правильное». Ниже понятие правильности переносится на отдельный контракт.

Определение 4. Пусть $V$ - сеть. Контракт $v \in V$ называется когерентным, если каждая сеть $U$ такая, что $U \simeq\{v\}$, стабильна снизу относительно $(x(V)-v)$ (т.е. взятых как исходные запасы).

Разница между правильными сетями и когерентными (состоят из когерентных контрактов) состоит в том, что в первом случае сеть устойчива относительно разрыва (частичного) любых договоров из сети, а во втором разрывается (частично) только один договор. В общем случае эти понятия не эквивалентны (см. пример 1).

На рис. 1 в системе координат потребителя $i \in I$ геометрически изображена типичная ситуация правильной сети договоров, а на рис. 2 - стабильная, но не когерентная и неправильная сеть. Различие состоит в том, что в первом случае весь параллелограмм договоров не пересекается с $\mathcal{P}_{i}\left(\bar{x}_{i}\right)$, а во втором - пересекается, хотя все вершины не принадлежат $\mathcal{P}_{i}\left(\bar{x}_{i}\right)$.

Следующее утверждение полностью характеризует когерентные контракты в выпуклых договорных экономиках.

Утверждение 1. Пусть $V$ - некоторая сеть договоров. Тогда договор $v \in V$ является когерентным тогда и только тогда, когда найдутся такие линейные функииональ $p_{i}^{v} \neq 0$, ито

$$
\left\langle p_{i}^{v}, \mathcal{P}_{i}\left(x_{i}(V)\right)\right\rangle>\left\langle p_{i}^{v}, x_{i}(V)\right\rangle^{5} \&\left\langle p_{i}^{v}, v_{i}\right\rangle \geq 0
$$

для каждого $i \in \mathcal{I}$. Более того, в гладкой модели условие (1) выполняется для $p_{i}^{v}=\operatorname{grad} u_{i}\left(x_{i}(V)\right) \neq 0, i \in \mathcal{I}$.

Доказательство, основанное на применении теоремы отделимости, опускается.

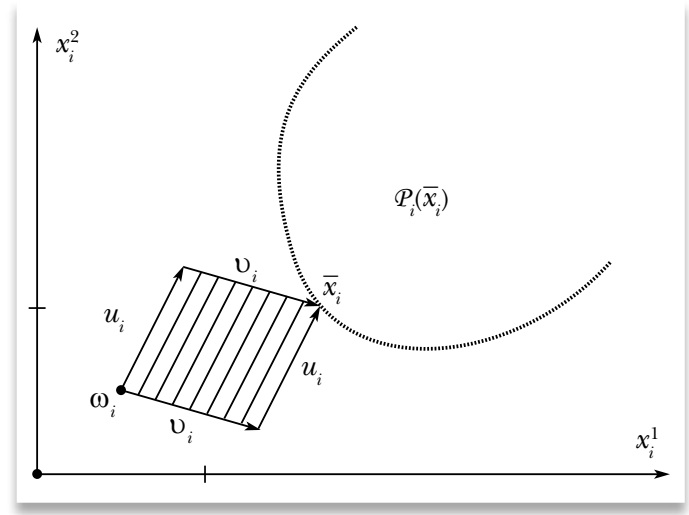

Pиc. 1

Сеть $\{u, v\}$ правильная

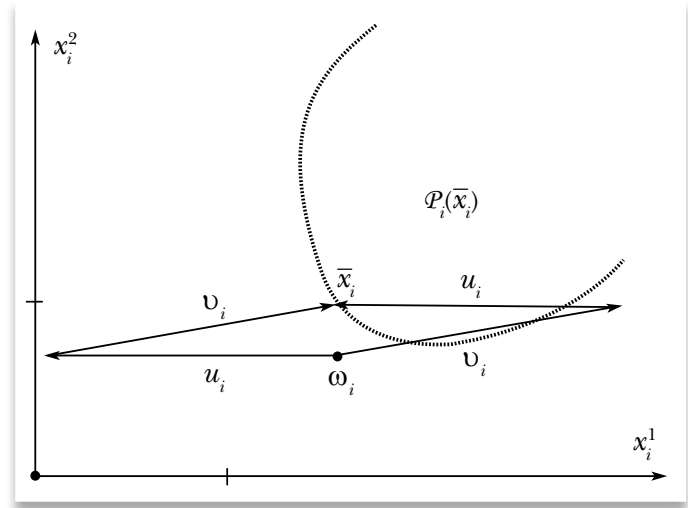

Pис. 2

Сеть $\{u, v\}$ неправильная

$\overline{{ }^{5} \text { Для } A, B} \subset E$ полагаем $\langle A, B\rangle=\{\langle a, b\rangle \mid a \in A, b \in B\}$, и $A>b$ означает, что $a>b$ для всех $a \in A$, симметрично для $\geq$. 
Следствие 1. Пусть $\mathcal{E}^{c}$ - гладкая договорная экономика. Тогда распределение $x$ является правильно договорным снизу тогда и только тогда, когда существует когерентная сеть $V$ такая, что $x=x(V)$. Более того, соотношение (1) истинно для каждого $v \in V$ при $p_{i}=p_{i}^{v}=\operatorname{grad} u_{i}\left(x_{i}(V)\right) \neq 0, i \in I$.

Следствие 1 , в частности, показывает, что для гладких экономик когерентные сети договоров устойчивы в отношении процедуры частичного разрыва (нескольких) договоров и, таким образом, совпадают с правильными сетями. При этом предположение о дифференцируемости функций полезности является существенным (соответствующие примеры легко строятся). Другое свойство правильных и когерентных контрактов состоит в том, что в гладкой экономике при сохранении агрегированных параметров обмена их можно замещать какой-либо другой правильной сетью договоров, получая при этом новую устойчивую снизу сеть. Это свойство также следует из утверждения 1.

В приложениях договорных моделей будет использоваться еще одно сильное свойство стабильности договоров - так называемые совершенные контракты. Чтобы ввести это понятие, рассмотрим еще один вид эквивалентности сетей договоров, определенной на множестве всех правильных сетей. Это (слабое) отношение эквивалентности можно определить следующим образом: пусть $U$ и $V$ некоторые правильные сети, тогда

$$
U \sim V \Leftrightarrow \sum_{u \in U} u=\sum_{v \in V} v
$$

Запись $U \sim V$ означает, что эти сети являются правильными и реализуют то же самое распределение. Также ясно, что $U \simeq V$ влечет $U \sim V$ для всех правильных сетей $U, V$. Если $V$ правильная сеть и $U$ другая правильная сеть такая, что $U \sim V$, то сеть $U$ будет называться виртуальной для сети $V$.

Определение 5. Распределение $x \in A(X)$ называется совершенно договорным, если существует правильная сеть $V$ такая, что $x=x(V)$ и для каждой правильной сети $U \sim V$ распределение $x=x(U)$ - договорное.

Концепцию совершенно договорного распределения можно трактовать как форму необязательного соглашения, а реализующую его сеть договоров как совокупность «протоколов о намерениях», являющуюся итогом предварительной стадии переговоров по обмену товарами. Это соглашение (распределение) защищено от потенциальной возможности того, что какая-либо коалиция окажется способной инициировать новый процесс перезаключения договоров. Члены коалиции исходят из того (могут надеяться), что в этом новом процессе им удастся повысить собственный уровень потребления (реализуя более предпочтительные потребительские программы). Сценарий может быть следующим. Через своих членов коа- 
лиция предлагает прочим агентам - не-членам коалиции, но вовлеченным в контракты с ее членами, переписать контракты на следующих условиях:

1) новое распределение ресурсов совпадает с текущим;

2) ни у кого не появится стимула частично разрывать новые контракты, т.е. у новой сети сохранится свойство устойчивости снизу относительно частичных разрывов договоров.

В таком случае не-члены коалиции могут пойти на это новое соглашение, так как у них нет явных побудительных мотивов для отказа (а возможно, члены коалиции являются убедительными переговорщиками). Но вот когда цель достигнута, коалиция рвет часть контрактов и заключает новый, что обеспечивает членам коалиции более предпочтительное потребление. Однако для совершенно договорного распределения это гипотетическое поведение не может быть выгодным ни для какой коалиции. Очевидно, что совершенно договорные распределения обладают более сильным типом стабильности.

Далее рассмотрим понятие нечетко договорного распределения. Понятие правильно договорного распределения предполагает, что агенты способны частично разрывать контракты так, что каждый контракт может быть разделен на несколько контрактов с теми же меновыми пропорциями, а затем часть этих контрактов может быть разорвана, т.е. вместо контракта $v \in V$ агенты будут иметь дело с контрактами $\left\{u_{\xi}\right\}$, удовлетворяющими $\sum u_{\xi}=v$ и $u_{\xi}=\lambda_{\xi} v$ для некоторого действительного $\lambda_{\xi} \geq 0$ для всех $\xi$. Таким образом, при частичном разрыве $v$ члены коалиции $S=\operatorname{supp}(v)$ должны координировать свои действия. Это координационное требование ослабляется для нечетко договорного распределения: агенты способны разрывать контракты асимметрично, вместе с $\sum u_{\xi}=v$ необходимо, чтобы $\left(u_{\xi}\right)_{i}=\lambda_{\xi i} v_{i}$ для некоторых действительных $\lambda_{\xi i} \geq 0 \quad \forall \xi, i$. Заметьте, что сейчас векторы $u_{\xi}$ даже могут не быть контрактами, так как условие $\sum_{i \in I} u_{\xi i}=0$ не обязано выполняться.

Пусть $V$ - сеть контрактов. Для каждого $v \in V$ рассмотрим и поставим в соответствие $n$-мерный вектор $t^{v}=\left(t_{1}^{v}, t_{2}^{v}, \ldots, t_{n}^{v}\right), 0 \leq t_{i}^{v} \leq 1 \quad \forall i \in I$, и пусть $v^{t}=\left(t_{1}^{v} v_{1}, t_{2}^{v} v_{2}, \ldots, t_{n}^{v} v_{n}\right)-$ кортеж продуктовых наборов, образованных из контракта $v=\left(v_{i}\right)_{i \in I}$ в случае, когда агенты «разрывают» индивидуальные наборы (фрагменты) этого контракта в долях $\left(1-t_{i}^{v}\right)_{i \in I}$. Обозначим $T(V)=T=\left\{t^{v} \mid v \in V\right\}$ и введем

$$
V^{T}=\left\{v^{t} \mid v \in V, t^{v} \in T\right\}, \Delta\left(V^{T}\right)=\sum_{v^{t} \in V^{T}} v^{t} .
$$

Определение 6. Распределение $x \in \mathcal{A}(X)$ называется нечетко договорным, если существует правильная сеть $V: x=x(V)$ и для каждого 
$T(V)$ распределение $x^{T}=\omega+\Delta\left(V^{T}\right)$ является договорным сверху.В экономических терминах это понятие можно пояснить следующим образом. В договорном процессе агенты могут делать ошибки, координация между членами коалиции может работать несовершенно и т.д. В результате агент $i$ может (ошибочно) считать, что после частичного разрыва текущих договоров он будет иметь продуктовый набор $x_{i}^{T}$ и что ресурсы из $x_{i}^{T}$ можно обменять в рамках нового (взаимовыгодного) контракта. Если распределение $x(V)$ не является нечетко договорным, тогда агент (потенциально) может разрушить соглашение и распределение изменится. Таким образом, нечетко договорные распределения защищены от данного способа, разрушающего договорные соглашения.

Заканчивая описание разных видов стабильности, которые могут применяться к распределению в контрактной экономике, предположим, что множество допустимых контрактов можно представить как (конечное) объединение звездных множеств, т.е. это множество имеет вид $\mathcal{W}=\bigcup_{\xi} V_{\xi}$. В частности, множества $V_{\xi}$ могут быть выпуклыми или быть подпространствами (как это имеет место в неполных рынках и моделях с дифференцированной информацией). В таком случае контракты из сети $V$ можно дифферениировать по признаку принадлежности к множествам $V_{\xi}$ (одному или нескольким) и потребовать, что если $v \in V_{\xi}$ для данного $\xi$, то контракт $v$ должен обладать одним из описанных свойств стабильности, т.е. является когерентным, правильным или совершенным, или ни тем и ни другим. Если такого рода соответствие установлено, то распределение $x(V)$ называется сложно договорным. Другими словами, сложно договорное распределение стабильно относительно процедур разрыва договоров соответствующего типа (в зависимости от множества, которому принадлежит контракт) и относительно заключения новых (допустимых) контрактов. Кроме того, могут существовать дополнительные требования в отношении сети, реализующей сложно договорное распределение: требования к совместной стабильности договоров (см., например, (Маракулин, 2003)).

\section{2. Договоры в стандартной экономике обмена}

В классической постановке модель экономики чистого обмена неявным образом предполагает, что между потребителями возможны любые обмены товарами. Единственное ограничение, накладываемое на реализованные потребительские программы (наборы), - это принадлежность к потребительским множествам, т.е. чтобы распределение было достижимо. Поэтому при пополнении модели обмена договорным механизмом логично считать, что допустимы любые договоры, т.е. предполагать $\mathcal{W}=L$, где $L$ - пространство состояний. 
В остальных отношениях стандартная модель полностью совпадает с моделью $\mathcal{E}$.

Далее напомним некоторые определения.

Пара $(x, p)$ называется квазиравновесиеммодели $\mathcal{E}$, если $x \in \mathcal{A}(X)$, $p \neq 0$ - линейный функционал над $E$ и $\left\langle p, \mathcal{P}_{i}\left(x_{i}\right)\right\rangle \geq p x_{i}=p \omega_{i} \quad \forall i \in \mathcal{I}$.

Квазиравновесие, такое что $x_{i}^{\prime} \in \mathcal{P}_{i}\left(x_{i}\right)$ влечет $p x_{i}^{\prime}>p x_{i}$, называется вальрасовским, или конкурентным, равновесием.

Говорят, что распределение $x \in \mathcal{A}(X)$ доминируется (блокируется по) коалицией (непустой) $\mathcal{S} \subset \mathcal{I}$, если существует такой $y^{S} \in \prod_{i \in S} X_{i}$, что $\sum_{i \in S} y_{i}^{S}=\sum_{i \in S} \omega_{i}$ и $y_{i}^{S} \in \mathcal{P}_{i}\left(x_{i}\right)$ для каждого $i \in \mathcal{S}$.

Ядро модели $\mathcal{E}$ - обозначенное как $\mathcal{C}(\mathcal{E})$ - это множество всех $x \in \mathcal{A}(X)$, которые не блокируются никакой коалицией.

Слабая гранииа Парето модели $\mathcal{E}$ - обозначение $\mathcal{P B}^{w}(\mathcal{E})$ - это множество таких $x \in \mathcal{A}(X)$, которые не блокируются коалицией $\mathcal{I}$ (всех агентов).

Распределение $x \in \mathcal{A}(X)$ называется индивидуально ращиональным, если не доминируется одноэлементными коалициями $(\mathcal{I R}(\mathcal{E})-$ множество всех таких распределений). Из определений следует, что $\mathcal{C}(E) \subset \mathcal{P B}^{w}(E) \cap \mathcal{I R}(\mathcal{E})$.

Сделаем некоторые замечания в отношении понятия оптимальности по Парето (границы Парето). В теории хорошо известна и более сильная концепция, называемая иногда сильной границей Парето. Для предпочтений $\succeq_{i}$, удовлетворяющих аксиомам предпоряд$\mathrm{ka}^{6}, x=\left(x_{i}\right)_{I} \in \mathcal{A}(X)$ сильно оптимально по Парето, если не существует $z=\left(z_{i}\right)_{I} \in \mathcal{A}(X)$, удовлетворяющего $z_{i} \succeq_{i} x_{i} \forall i \in \mathcal{I} \quad \& \quad \exists j \in \mathcal{I}: z_{j} \succ_{j} x_{j}$.

Обозначим $\mathcal{P} \mathcal{B}^{s}(\mathcal{E})$ сильную границу Парето (множество всех сильно оптимальных по Парето распределений). Из определений следует $\mathcal{P} \mathcal{B}^{s}(\mathcal{E}) \subset \mathcal{P B}^{w}(\mathcal{E})$. Теоретически имеется еще одна возможность определения понятия оптимальности в модели экономики, занимающая промежуточное место между слабой и сильной оптимальностью по Парето. Именно такого рода оптимальность реализуется в договорных сверху распределениях.

Назовем распределение $x=\left(x_{i}\right)_{I} \in \mathcal{A}(X)$ строго оптимальным nо Парето, если не существует коалиции $\mathcal{S} \subset \mathcal{I}$, для которой найдется $y^{S} \in \prod_{i \in S} X_{i}$ такой, что $\sum_{i \in S} y_{i}^{S}=\sum_{i \in S} x_{i}$ и $y_{i}^{S} \succ_{i} x_{i}$ для каждого $i \in S$. Другими словами, $x$ является распределением из ядра в экономике, совпадающей с исходной моделью с единственным отличием, - именно это распределение принимается в качестве исходного. Пусть $\mathcal{P B}(\mathcal{E})$ - строгая граница Парето. Из определений следует, что $\mathcal{P} \mathcal{B}^{s}(\mathcal{E}) \subset \mathcal{P B}(\mathcal{E}) \subset \mathcal{P} \mathcal{B}^{w}(\mathcal{E})$. Следовательно, если некоторый $x=\left(x_{i}\right)_{I} \in \mathcal{P} \mathcal{B}^{w}(\mathcal{E})$ и при этом условия такие, что можно показать, что 
$x$ сильно оптимален по Парето (например, если предпочтения локально ненасыщенные и $x \in \operatorname{int} X)^{7}$, то это распределение будет и строго оптимальным по Парето.

Другое важное понятие, плодотворно работающее в теории экономического равновесия, - это концепция нечеткого ядра. Напомним, что любой вектор $t=\left(t_{1}, \ldots, t_{n}\right) \neq 0, \quad 0 \leq t_{i} \leq 1 \quad \forall i \in \mathcal{I}$ отождествляется с нечеткой коалицией, где вещественные $t_{i}$ интерпретируются как мера участия потребителя $i$ в данной коалиции. Говорят, что коалиция $t$ доминирует (блокирует) распределение $x \in A(X)$, если найдется такой $y^{t} \in \prod_{I} X_{i}$, что

и при этом

$$
\sum_{i \in I} t_{i} y_{i}^{t}=\sum_{i \in I} t_{i} \omega_{i} \Leftrightarrow \sum_{i \in I} t_{i}\left(y_{i}^{t}-\omega_{i}\right)=0
$$

$$
y_{i}^{t} \succ_{i} x_{i}, \quad \forall i \in \operatorname{supp}(t)=\left\{i \in I \mid t_{i}>0\right\} .
$$

Множество всех неблокируемых по нечетким коалициям достижимых состояний, обозначенное как $C^{f}(E)$, называется нечетким ядром модели $\mathcal{E}$.

Следующая теорема (доказательство опущено) характеризует распределения из ядра и других описанных множеств в терминах (разного вида) договорных распределений.

Теорема 1. Пусть $\mathcal{W}=L$ для договорной экономики $\mathcal{E}^{c}$, тогда распределение $x$ :
i) договорное $\Leftrightarrow x \in \mathcal{C}(\mathcal{E}) \cap \mathcal{P B}(\mathcal{E})$;
ii) договорное сверху $\Leftrightarrow x \in \mathcal{P B}(\mathcal{E})$;
iii) договорное снизу $\Leftrightarrow x \in \mathcal{I} \mathcal{R}(\mathcal{E})$;
iv)слабо договорное $\Leftrightarrow x \in \operatorname{IIR}(\mathcal{E}) \cap \mathcal{P B}(\mathcal{E})$.

Далее охарактеризуем равновесные распределения в правильно договорных терминах. Результат следующей теоремы позволяет рассматривать стабильность распределения относительно частичного разрыва договоров как специфическую форму условий совершенной конкуренции, что представляет иной, договорной взгляд на этот предмет. Развитием этого подхода является идея нечетко договорного распределения, чья взаимосвязь с равновесиями (совпадают при более слабых предположениях) будет раскрыта в дальнейшем анализе.

Теорема 2. Пусть $\mathcal{E}^{c}$ гладкая договорная экономика $u \quad x \in \operatorname{int} X$ достижимое распределение такое, ито $\operatorname{grad} u_{i}\left(x_{i}\right) \neq 0$ для некоторого $i$ и все агенты ненасыщенные. Тогда следующие утверждения эквивалентны:

i) $x$ равновесное распределение;

ii) $x$ оптимально по Парето и существует когерентная сеть $V$, реализующая это распределение, т.е. $x=x(V)$, сеть $V$ когерентная и устойчивая сверху;

iii) $x$ правильно договорное распределение;

iv) $x$ совершенно договорное распределение.

\footnotetext{
${ }^{7}$ Кроме того, известно, что если предпочтения строго монотонны и $X_{i}=\mathbb{R}_{+}^{l}$ ( $l$ - число продуктов) для всех $i$, то понятия сильной и слабой оптимальности по Парето совпадают.
} 
Более того, если $(x, p)$ - равновесие и $V$ - некоторая сеть, реализующал $x=x(V)$, то $V-$ когерентная сеть тогда и только тогда, когда $р v_{i}=0$ $\forall v \in V, \forall i \in \mathcal{I}$.

Несложный анализ показывает, что импликация $(i) \Rightarrow($ iii $)$ имеет место в общем случае - для негладких предпочтений и без требования $x \in \operatorname{int} X$, т.е. в стандартной модели обмена каждое равновесие является правильно договорным. Прочие элементы доказательства можно найти в (Маракулин, 2003).

Далее рассмотрим пример, демонстрирующий различие в понятиях договорного распределения разного типа. Конечно, чтобы это различие проявилось при допущении частичного разрыва договоров, необходимо, чтобы нарушились условия теоремы 2 - либо полезности должны быть негладкими, либо распределение принадлежит границе множества $X$. Этот пример, принадлежащий А.Н. Козыреву (1982), показывает, что для недифференцируемых функций полезности правильно договорное распределение может не быть равновесным.

Пример 1. Рассмотрим двухпродуктовую модель экономики чистого обмена с двумя потребителями, в которой $X_{i}=\mathbb{R}_{+}^{2}$, а предпочтения задаются строго монотонными функциями полезности, определенными на $\mathbb{R}_{+}^{2}$ и имеющими вид:

$$
u_{1}\left(x_{1}, x_{2}\right)=2 \sqrt{x_{1} x_{2}}+x_{1}+x_{2}, \quad u_{2}\left(y_{1}, y_{2}\right)=2 \sqrt{y_{1} y_{2}}+y_{1}+y_{2}+\min \left\{y_{1}, y_{2}\right\} .
$$

Примем $\omega_{1}=(1,0), \omega_{2}=(0,1), \bar{\omega}=(1,1), \omega=\left(\omega_{1}, \omega_{2}\right)=((1,0),(0,1))$ в качестве исходных запасов и будем рассуждать в пределах «ящика Эджворта»: $E B(\bar{\omega})=\left\{x \in \mathbb{R}^{2} \mid 0 \leq x \leq(1,1)=\bar{\omega}\right\}, \quad$ точки которого $x \in E B(\bar{\omega})$ можно понимать как потребление агента $1,(\bar{\omega}-x)$ - как потребление агента 2 , и, наконец, их можно ассоциировать с распределением продуктов $(x, \bar{\omega}-x)$.

Соображения симметрии и непосредственный анализ показывают, что границей Парето в данном примере является диагональ в $E B(\bar{\omega})$ :

$$
\mathcal{P B}=\operatorname{co}\{(0,0),(1,1)\}=\left\{x \in E B(\bar{\omega}) \mid x_{1}=x_{2}=\alpha, 0 \leq \alpha \leq 1\right\} .
$$

Так как равновесное распределение оптимально по Парето и если это внутренняя точка из ящика, то с точностью до нормировки вектор равновесных цен должен совпадать с $\operatorname{grad} u_{1}\left(x_{1}\right)$ и, следовательно, равновесными ценами должен быть вектор $(1,1)$. Точки $(1,1)$ и $(0,0)$ не являются равновесными распределениями, поэтому $p=(1,1)$ - единственный (с точностью до нормировки) вектор равновеснъх иен. Используя бюджетные равенства $p x_{i}=p \omega_{i}$, находим (единственное) равновесное распределение, которому в ящике Эджворта отвечает вектор потребления первого агента $(1 / 2,1 / 2)$.

Ядро в экономике с двумя потребителями совпадает с множеством $\mathcal{P B} \cap \mathcal{I R}$, которое, в свою очередь, является множеством всех договорных и слабо договорных распределений и может быть легко вычислено: 


$$
\begin{gathered}
\mathcal{P B} \cap \mathcal{I R}=\left\{x \in \mathcal{P B} \mid u_{1}(x) \geq u_{1}\left(\omega_{1}\right), u_{2}(\bar{\omega}-x) \geq u_{2}\left(\omega_{2}\right)\right\}= \\
=\operatorname{co}\{(1 / 4,1 / 4),(4 / 5,4 / 5)\} .
\end{gathered}
$$

Найдем множество правильно договорных распределений. Его можно описать как множество всех таких точек $x=(\alpha, \alpha)$ из $\mathcal{P B} \subset \mathcal{E B}$, для которых производные функций полезности $u_{1}$ и $u_{2}$ неположительны по направлениям $h_{1}=\omega_{1}-(\alpha, \alpha)$ и $h_{2}=\omega_{2}-(1-\alpha, 1-\alpha)$, т.е. необходимо решить систему неравенств

$$
\begin{aligned}
& \partial_{h_{1}} u_{1}(\alpha, \alpha) \leq 0, \\
& \partial_{h_{2}} u_{2}(1-\alpha, 1-\alpha) \leq 0 .
\end{aligned}
$$

Непосредственное вычисление дает $\operatorname{grad} u_{1}(\alpha, \beta)=(\sqrt{\beta / \alpha}+1, \sqrt{\alpha / \beta}+1)$ для всех $\alpha>0, \beta>0$ и $\operatorname{grad} u_{2}(1-\alpha, 1-\beta)=(\sqrt{(1-\beta) /(1-\alpha)}+2, \sqrt{(1-\alpha) /(1-\beta)}+1)$ при $\alpha>\beta>0,1-\alpha>0$, откуда после нахождения скалярных произведений и подстановки $\alpha=\beta$ (т.е. переходя к пределу при $\beta \rightarrow \alpha$ ) получаем

$$
\begin{aligned}
& 1-2 \alpha \leq 0, \\
& 5 \alpha-3 \leq 0 .
\end{aligned}
$$

В итоге множество всех правильно договорных распределений описывается как со $\{(1 / 2,1 / 2),(3 / 5,3 / 5)\}$ и не совпадает с множеством равновесных распределений (но содержит его!). Ситуацию иллюстрирует рис. 3.

В рамках этого примера можно также выявить структуру множества распределений правильно договорных снизу. Любопытно, что здесь это множество не выпуклое (данный анализ см. в (Маракулин, 2003)).

На этом примере можно продемонстрировать различие между когерентной и правильной сетью. Действительно, рассмотрим новое исходное распределение $\omega^{\varepsilon}=\omega-\varepsilon w$ при достаточно малом $\varepsilon>0$ и сеть договоров $V^{\varepsilon}=\{v, \varepsilon w\}$, где $v=\left(v_{1}, v_{2}\right), v_{1}=-v_{2}=(3 / 5,3 / 5)-$

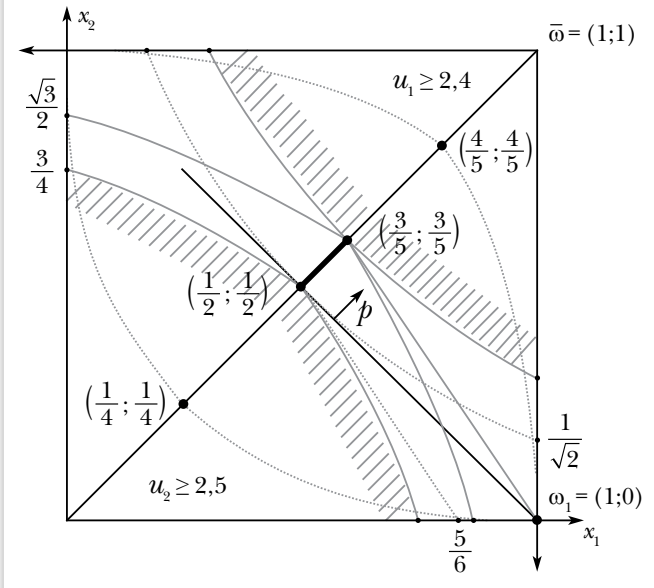

Pис. 3

Негладкие предпочтения
$-(1,0)=(-2 / 5,3 / 5)$ и $w=\left(w_{1}, w_{2}\right), w_{1}=$ $=-w_{2}=(3,-2)-$ договор, в котором потребитель 1 обменивает две единицы продукта 2 на три единицы продукта 1. Здесь можно, например, положить $\varepsilon=1 / 6$. Очевидно, что $\omega^{\varepsilon}+v+\varepsilon w=((3 / 5,3 / 5),(2 / 5,2 / 5))$, т.е. рассмотренное распределение реализуется сетью $V^{\varepsilon}$ относительно запасов $\omega^{\varepsilon}$. Аккуратные вычисления производных по направлению показывают, что каждый договор сети $V^{\varepsilon}$ является когерентным относительно $\omega^{\varepsilon}$. Однако для каждого $\varepsilon \in(0 ; 1 / 6]$ 
сеть $V^{\varepsilon}$ не является правильной, так как после разрыва половины договора $\varepsilon w$ и частичного разрыва договора $v$ в объеме $\delta=3 \varepsilon / 2<1$ реализуется распределение, в котором потребительская программа участника 1 имеет вид

$$
\begin{aligned}
\varepsilon w_{1} / 2+(1-5 \varepsilon / 2) v_{1}+\omega_{1}^{\varepsilon} & =\varepsilon(3,-2) / 2+(1-3 \varepsilon / 2)(-2 / 5,3 / 5)+(1,0)-\varepsilon(3,-2)= \\
& =(3 / 5,3 / 5)-\varepsilon(1 / 2,1 / 2)=x_{1}^{\varepsilon} .
\end{aligned}
$$

Следовательно, предложенный частичный разрыв договоров оказывается выгодным для участника 2, так как при этом он увеличивает свое потребление на $\varepsilon(1 / 2,1 / 2)$, а значит, сеть $V^{\varepsilon}$ не является правильной относительно $\omega^{\varepsilon}$.

Представленный пример достаточно поучительный и стимулирует более тщательное изучение математических свойств правильнодоговорных, а также совершенно-договорных распределений разного рода в ситуациях, когда нарушены условия теоремы 2. Однако в данной работе это исследование опущено. Перейдем далее к анализу нечетно договорных распределений.

Начнем с рассмотрения характеристических свойств распределений из нечеткого ядра. Элементы нечеткого ядра задаются соотношениями (3), (4), которые для ненасыщенных предпочтений можно переписать в эквивалентном виде $0 \in \sum_{i \in I} t_{i}\left(\mathcal{P}_{i}\left(x_{i}\right)-\omega_{i}\right)$. Таким образом, в этом случае условие $x \in \mathcal{C}^{f}(\mathcal{E})$ эквивалентно 8

$$
0 \notin \operatorname{co}\left[\bigcup_{I}\left(\mathcal{P}_{i}\left(x_{i}\right)-\omega_{i}\right)\right]
$$

откуда, в частности (после применения теоремы отделимости), следует, что элементы нечеткого ядра являются квазиравновесными. Ниже мы дадим другое важное описание, данное в «геометрических» терминах. С этой целью рассмотрим множества $\Omega_{i}\left(x_{i}\right)=\operatorname{co}\left(\mathcal{P}_{i}\left(x_{i}\right) \cup\left\{\omega_{i}\right\}\right), i \in \mathcal{I}$. В силу выпуклости $\mathcal{P}_{i}\left(x_{i}\right)$, для $\mathcal{P}_{i}\left(x_{i}\right) \not \varnothing$ имеем

$$
\operatorname{co}\left(\mathcal{P}_{i}\left(x_{i}\right) \cup\left\{\omega_{i}\right\}\right)=\bigcup_{0 \leq \lambda \leq 1}\left[\lambda \mathcal{P}_{i}\left(x_{i}\right)+(1-\lambda) \omega_{i}\right]=\bigcup_{0 \leq \lambda \leq 1} \lambda\left(\mathcal{P}_{i}\left(x_{i}\right)-\omega_{i}\right)+\omega_{i}, i \in \mathcal{I} .
$$

Отсюда следует, что условие $z+\omega \in \prod_{I} \Omega_{i}\left(x_{i}\right)$, где $\omega=\left(\omega_{1}, \ldots, \omega_{n}\right)$, эквивалентно существованию таких $0 \leq \lambda_{i} \leq 1$ и $\left[y_{i} \in \mathcal{P}_{i}\left(x_{i}\right) \neq \varnothing\right.$ и $y_{i}=\omega_{i}$ при $\left.\mathcal{P}_{i}\left(x_{i}\right)=\varnothing\right], i \in \mathcal{I}$, что $z=\left(\lambda_{1}\left(y_{1}-\omega_{1}\right), \ldots, \lambda_{n}\left(y_{n}-\omega_{n}\right)\right)$. Таким образом, в силу (3), (4) можно утверждать, что

$$
\begin{gathered}
x \in \mathcal{C}^{f}(\mathcal{E}) \Leftrightarrow \nexists z \in E^{I}, \quad z \neq 0: z+\omega \in \prod_{I} \Omega_{i}\left(x_{i}\right) \& \sum_{i \in I} z_{i}=0 \Leftrightarrow \\
\Leftrightarrow \prod_{I} \Omega_{i}\left(x_{i}\right) \bigcap\left\{\left(z_{1}, \ldots, z_{n}\right) \in E^{I} \mid \sum_{i \in I} z_{i}=\sum_{i \in I} \omega_{i}\right\}=\{\omega\} .
\end{gathered}
$$

Характеризация последней строки в формуле (6) имеет место и для насыщенных предпочтений. Таким образом доказана следующая

\footnotetext{
${ }^{8}$ Так как доминирование по произвольным нечетким коалициям эквивалентно доминированию по нормированным коалициям.
} 
Лемма 1. Распределение $x \in \mathcal{A}(X)$ принадлежит нечеткому ядру тогда и только тогда, когда выполнено соотношение (6).

Далее мы намерены проявить специфику распределений из нечеткого ядра в чисто договорных категориях. Начнем с предварительного результата, описывающего математические свойства нечетко договорных распределений, что имеет также и самостоятельный интерес.

Лемма 2. Правильно-договорное снизу распределение $x \in \mathcal{A}(X)$ является нечетко договорным тогда и только тогда, когда

$\prod_{I}\left[\left(P_{i}\left(x_{i}\right)+\operatorname{co}\left\{0, \omega_{i}-x_{i}\right\}\right) \cup\left\{\omega_{i}\right\}\right] \bigcap\left\{\left(z_{1}, \ldots, z_{n}\right) \in E^{I} \mid \sum_{i \in I} z_{i}=\sum_{i \in I} \omega_{i}\right\}=\{\omega\}$.

Постольку поскольку для каждого достижимого $x=\left(x_{i}\right)_{I}$ имеем $\omega_{i} \in \Omega_{i}\left(x_{i}\right) \subset\left(P_{i}\left(x_{i}\right)+\operatorname{co}\left\{0, \omega_{i}-x_{i}\right\}\right) \cup\left\{\omega_{i}\right\}, \forall i \in I$, то в силу лемм 1,2 каждое нечетко договорное распределение принадлежит нечеткому ядру экономики. Однако свойство распределения быть нечетко договорным все еще немножко сильнее, нежели факт принадлежности нечеткому ядру. Следующий результат проясняет соотношения между двумя нечеткими понятиями.

Лемма 3. Пусть $x \in \mathcal{A}(X)$ u $\mathcal{P}_{i}\left(x_{i}\right) \not \neq \varnothing$ для всех $i \in I$. Тогда $x \in \mathcal{C}^{f}(\mathcal{E})$ влечет:

$$
\prod_{I}\left(\mathcal{P}_{i}(x)+\operatorname{co}\left\{0, \omega_{i}-x_{i}\right\}\right) \bigcap\left\{\left(z_{1}, \ldots, z_{n}\right) \in E^{I} \mid \sum_{i \in I} z_{i}=\sum_{i \in I} \omega_{i}\right\}=\varnothing .
$$

Доказательства лемм приведены в (Marakulin, 2006). Сравнение формул (8) и (7) проясняет различие между распределениями из нечеткого ядра и нечетко договорными. Это различие не слишком велико, что позволяет интерпретировать распределения из нечеткого ядра как нечетко договорные 9 . В итоге как следствие лемм 1-3 можно сформулировать теорему.

Теорема 3. Если модель такова, ито каждое нетривиальное квазиравновесие является равновесием ${ }^{10}$, то каждый элемент нечеткого ядра является нечетко договорным распределением и, следовательно, эти нечеткие кониепиии совпадают между собой и равновесием.

Итак, в работе представлены базовые элементы теории бартерных контрактов, развитые для экономики обмена. Здесь рассматривается ряд концепций стабильности договорного распределения и сетей договоров и выясняются их взаимосвязи с понятиями, известными в классической теории. Тем самым равновесие, граница Парето, ядро, нечеткое ядро получают описание в договорных категориях. Показано, что договорной подход - это довольно удобный инструмент для моделирования условий совершенной конкуренции и представляет ее альтернативные виды. Таким образом, договорной подход естественным образом дополняет классический и может плодотворно работать в исследованиях современных неклассических моделей.

\footnotetext{
${ }^{9}$ В литературе известна интерпретация элементов нечеткого ядра как равновесий Эджворта, однако сами эти элементы всегда служили скорее техническим инструментом, нежели содержательным понятием.

${ }^{10}$ Условия, обеспечивающие этот факт, необременительные и хорошо известны в литературе, например, это может быть нередуцируемость.
} 


\section{Литература}

Козырев А.Н. (1981): Устойчивые системы договоров в экономике чистого обмена / / Оптимизаиия. Вып. 29 (44): С. 66-78.

Козырев А.Н. (1982): Договорные и вполне договорные состояния в абстрактной экономике. Новосибирск: Институт математики СО АН СССР. Препринт № 7.

Макаров В.Л. (1980): О понятии договора в абстрактной экономике / / Оптимизаиия. Вып. 24(41). С. 5-17.

Макаров В.Л. (1982): Экономическое равновесие: существование и экстремальные свойства / / Итоги науки и техники: Современные проблемы математики. Т. 19. С. 23-58.

Маракулин В.М. (2003): Договоры и коалиционное доминирование в неполных рынках // Консорииум экономических исследований и образования. Серия «Научные доклады». № 02/04.

Маракулин В.М. (2006): О сходимости договорных траекторий в экономике чистого обмена / / Консорииум экономических исследований и образования. Серия «Научные доклады». № 06/07.

Маракулин B.М. (2009): Экономики с асимметрично информированными агентами: концепция предельной информации / / Журнал Новой экономической ассочиачии. Т. 1-2. С. 62-85.

Полтерович В.М. (1970): Математические модели перераспределения ресурсов. М.: ЦЭМИ.

Aliprantis C. (ed.) (2001): Economic Theory. Vol. 18. Berlin/Heidelberg: SpringerVerlag.

Anderson R.M. (1992): Non-Standard Analysis with Applications to Economics. In: Hildenbrand W., Sonnenschein H. (eds.) «Handbook of Mathematical Economics». Vol. IV. Amsterdam: North-Holland. P. 2145-2208.

Arrow K.J., Hahn F.H. (1991): General Competitive Analysis. Amsterdam, N.Y., Oxford, Tokyo: North-Holland.

Aumann R. J. (1964): Market with a Continuum of traders // Econometrica. Vol. 32. P. 39-50.

Dagan N., Serrano R., Volij O. (2000): Bargaining, Coalitions and Competition// Economic Theory. Vol. 15(2). P. 279-296

Florenzano M., Marakulin V.M. (2001): Production Equilibria in Vector Lattices / / Econ. Theory. Vol. 17(3). P. 577-598.

Glycopantis D., Yannelis N.C. (eds.) (2004): Differential Information Economies (Collected Papers): Berlin/Heidelberg: Springer-Verlag.

Gale D. (1978): The Core of a Monetary Economy without Trust / / J. Econ. Theory. Vol. 19. P. 456-491.

Gale D. (2000): Strategic Foundations of General Equilibrium - Dynamical Matching and Bargaining Games. Cambrige: Cambrige University Press.

Graham D.A., Jennergren L.P., Peterson D.P. et al. (1976): Trader-commodity Parity Theorems / / J. Econom. Theory. Vol. 12. P. 443-454.

Hildenbrand W. (1974): Core and Equilibria of a Large Economy. Princeton: Princeton University Press. 
Madden P.J. (1975). Efficient Sequences of Non-Monetary Exchange / / Rev. Econ. Studies. Vol. 42. P. 581-595.

Magill M., Quinzii M. (2002). Theory of Incomplete Markets. Cambridge, Massachusetts, London: The MIT Press.

Marakulin V.M. (2006). Equilibrium Analysis in Kantorovich Spaces // Journal of Mathematical Sciences. Vol. 133(4). P. 1477-1490.

Muthoo A. (1999). Bargaining Theory with Applications. Cambridge: Cambridge University Press.

Repullo R. (1988). The Core of an Economy with Transactions Costs / / Rev. Econ. Studies. Vol. 55. P. 447-458.

Schwable U. (1999). The Core of Economies with Asymmetric Information. Berlin/ Heidelberg/N.Y.: Springer-Verlag.

Vind K. (1995). Perfect Competition or the Core // European Econ. Rev. Vol. 39. P. 1733-1745.

Wilson R. (1978). Information, Efficiency, and the Core of an Economy // Econometrica. Vol. 46. P. 807-816.

Yildiz M. (2003). Walrasian Bargaining / / Games and Economic Behavior. Vol. 45(2). P. 465-487.

Поступила в редакиию 5 ноября 2009 г.

\section{V.M. Marakulin}

Sobolev Institute of Mathematics, Russian Academy of Sciences, Novosibirsk

\section{Contracts and Domination in Competitive Economies}

A new concept of contract-based domination by coalitions for competitive economies is proposed and studied in the paper. This concept is based on the notion of (barter) contract (an elementary exchange of commodities). Here classical coalitions domination is transferred onto systems (webs) of contracts and this way implemented contractual allocations which stability properties are investigated. It is shown that suggested approach is efficiently modeling perfect competition conditions and allows to describe various known classical concepts for a perfect economy - equilibria, core, fuzzy core etc. - in pure game-theoretical terms. For non-perfect economies, in which not every contract is permissible, it may serve as one of the model primitives to refine and to solve various theoretical problems.

Keywords: exchange economy, contract, contractual allocation, competitive equilibrium, core.

JEL Classification: C62, D51. 